\title{
Características clínicas, demográficas y acceso a los productos sin gluten de pacientes con enfermedad celíaca registrados en la FUPACEL
}

\author{
Karina Gisselle Román-Giménez (iD), Elsi Fiorella Cuevas-Duarte iD, Lilian Acosta \\ Sanchez iD, * Margarita Samudio \\ ${ }^{1}$ Universidad del Pacifico. Facultad de Ciencias Médicas. Asunción, Paraguay
}

Cómo referenciar este artículo/

How to reference this article:

\begin{abstract}
Román Giménez KG, Cuevas Duarte EF, Acosta L, Samudio M. Características clínicas, demográficas y acceso a los productos sin gluten de pacientes con enfermedad celíaca registrados en la FUPACEL. Mem. Inst. Investig. Cienc. Salud. 2021; 19(2): 78-85
\end{abstract}

\section{RE S U ME N}

La enfermedad celíaca (EC) es una enfermedad inflamatoria autoinmune que afecta la mucosa del intestino delgado que lleva a un estado de mal absorción en pacientes genéticamente susceptibles desencadenada por la ingesta de gluten. Tiene síntomas inespecíficos, complicaciones asociadas y no existe a nivel nacional una ley de apoyo económico para las personas con dificultad para seguir una dieta libre de gluten. EI objetivo de este estudio observacional, descriptivo, de corte transversal fue describir las características clínicas, demográficas y acceso a productos sin gluten de 237 pacientes con enfermedad celíaca registrados en la Fundación Paraguaya de Celiacos (FUPACEL) en el 2020 y que aceptaron responder una encuesta en línea publicada en la página oficial de la FUPACEL. El 70,4\% de los participantes era del sexo femenino, la edad de diagnóstico fue en el 34,5\% entre 20-30 años, $48 \%$ tenía algún familiar con EC, el $69,1 \%$ presentó distensión abdominal como sintomatología inicial, $16,8 \%$ presentó hipotiroidismo como patología asociada, y $42 \%$ fue hospitalizado al menos una vez. El $82,7 \%$ de los encuestados respondió que la dieta era suficiente como tratamiento, $48 \%$ tenía un trabajo y podía solventar los gastos, sin embargo, el $79,3 \%$ opinó tener acceso limitado a los productos sin gluten, el $92 \%$ que los alimentos sin gluten eran costosos y que se necesitaba una ley de apoyo económico para solventar los gastos. La mayoría reconoció que la dieta sin gluten como tratamiento era suficiente, sin embargo, por su alto costo se necesita una ley de apoyo económico para las personas con enfermedad celiaca.

Palabras clave: Enfermedad celíaca, presentación clínica, dieta libre de gluten.

\section{Clinical and demographic characteristics, and access to gluten free products of patients with celiac disease registered in the FUPACEL}

\begin{abstract}
Celiac disease (CD) is an autoimmune inflammatory disease that affects the mucosa of the small intestine that leads to a state of malabsorption in genetically susceptible patients triggered by the ingestion of gluten. It has nonspecific symptoms, associated complications, and there is no national financial support law for people with difficulty following a gluten-free diet. The objective of this observational, descriptive, crosssectional study was to describe the clinical, demographic characteristics and access to gluten-free products of 237 patients with celiac disease registered in the FUPACEL in
\end{abstract}

Fecha de recepción: Junio 2021. Fecha de aceptación: Julio 2021

* Autor correspondiente: Margarita Samudio. Universidad del Pacifico. Facultad de Ciencias Médicas. Asunción, Paraguay. Email: margarita.samudio@gmail.com 
2020 who accepted to answer a online survey published in the official FUPACEL webpage. Seventy-point four percent of the patients were female, their age of diagnosis was between $20-30$ years in $34.5 \%$, 48\% had relatives with CD, $69.1 \%$ presented abdominal distension as initial symptoms, hypothyroidism was present in $16.8 \%$ as associated pathology, and $42 \%$ were hospitalized at least once. Eighty-twopoint seven percent of those surveyed answered that diet was sufficient as a treatment, $48 \%$ had a job and could afford expenses, however, $79.3 \%$ said they had limited access to gluten-free products, $92 \%$ that gluten-free foods were expensive and a financial support law was needed to cover the costs. Most recognized that a glutenfree diet as a treatment was sufficient, however, due to its high cost, a law of financial support is needed for people with celiac disease.

Keywords: Celiac disease, clinical presentation, gluten-free diet.

\section{NTRODUCCIÓN}

La enfermedad celíaca (EC) es una enfermedad inflamatoria de origen autoinmune que afecta la mucosa del intestino delgado en personas genéticamente susceptibles y cuyo desencadenante es la ingesta de gluten. Es producida por la interacción de ciertos haplotipos (genes) del huésped que aportan la predisposición genética ${ }^{(1)}$ y el ambiente, que entrega las prolaminas del gluten en la dieta ${ }^{(2)}$. Otros factores, como la epigenética y la microbiota del huésped, participarían en la patogenia de la EC a través de mecanismos poco conocidos aún ${ }^{(3)}$. Se la reconoce como una condición autoinmune de alta frecuencia, que afecta a todas las edades ${ }^{(4)}$, con una prevalencia global de $1-3 \%$ de la población, en general es más frecuente en las mujeres ${ }^{(5-10)}$.

En una encuesta en línea a 1.058 personas en Paraguay se estimó la prevalencia de trastornos relacionado al gluten y la adherencia a una dieta libre de gluten (DLG) en la población adulta paraguaya ${ }^{(11)}$. La sensibilidad al gluten (SG) fue informada por el 10,30\% (8,53-12,29); SG no celíaco, 5,19\% (3,94-6,71); enfermedad celíaca diagnosticada por un médico, 3,11\% (2,15-4,35); alergia al trigo, 2,07\% (1,30-3,13); y adherencia a la DLG, $15,69 \%(13,55-18,02)$. Se estima que en Paraguay hay 60.000 celiacos, en base a una estimación de $1 \%$ de la población según los registros de la Fundación Paraguaya de Celiacos (FUPACEL) ${ }^{(12)}$.

La EC se diagnostica mediante la medición de anticuerpos antitransglutaminasa, antiendomisio o péptidos de gliadina deamidada y confirmación histológica en biopsia duodenal, que muestra diversos grados de infiltración linfocítica, hiperplasia de las criptas y aplanamiento de vellosidades ${ }^{(1)}$.

No existen muchos estudios a nivel nacional que caracterizen a los pacientes con EC. Así, en el 2011, se describió las características clínicas y demográficas de 656 adultos y niños registrados en la FUPACEL entre 1995 y octubre 2011 y que al momento del diagnóstico presentaba cuadro clínico, serología específica y biopsia duodenal compatibles con EC. La edad media fue $25 \pm 18$ años y predominó el sexo femenino $(72,4 \%)$, las formas familiares fue en $17,6 \%$, la diarrea crónica fue el motivo de consulta más frecuente $(62,7 \%)$, las enfermedades asociadas más frecuentes fueron la dermatitis herpetiforme $(33,8 \%)$ y la tiroiditis crónica autoinmune $(6,6 \%)^{(13)}$. En otro estudio que determinó la calidad de vida de 114 pacientes adolescentes y adultos del Paraguay portadores de la enfermedad celiaca con al menos 6 meses en dieta sin gluten que concurrieron a la FUPACEL y al Hospital Nacional de Itagua en el 2016, también hubo predominio del sexo femenino (58\%), la edad media de inicio de los síntomas fue de $18 \pm 10$ años (rango 1-49 años), y la edad media de diagnóstico fue $21 \pm 10$ años (rango 1-57 años) ${ }^{(14)}$.

Hasta hoy, el único tratamiento efectivo para la EC es la DLG, estricta, permanente y por toda la vida. Esta dieta elimina de la alimentación el trigo, centeno y cebada, incluidos tanto en los alimentos naturales como procesados ${ }^{(15)}$. Habitualmente la avena también se elimina por tener alta probabilidad de estar contaminada ${ }^{(16)}$. Sin embargo, la dieta sin gluten es compleja, costosa, restrictiva y difícil de cumplir ${ }^{(17)}$. Una encuesta realizada en España a personas con celiaquía y sus familiares encontró que el $90 \%$ de los encuestados consideran que la dieta sin gluten supone un 
sobrecosto superior al $20 \%$ en el precio a abonar por productos de consumo básico y el $91 \%$ tiene como preocupación el precio ${ }^{(18-19)}$.

Debido a la limitada información sobre las características y las limitaciones de las personas afectadas por esta enfermedad a la DLG, el objetivo de este estudio es describir las características clínicas, demográficas y acceso a productos sin gluten de pacientes con enfermedad celíaca registrados en la FUPACEL en el 2020.

\section{MATERI ALES Y MÉTODOS}

Diseño y lugar de estudio: observacional descriptivo, de corte transversal. FUPACEL es una fundación sin fines de lucro con el principal objetivo de conseguir una mejor calidad de vida para todos los celiacos del país. Además, promueve el desenvolvimiento del celiaco en la vida comunitaria, informa sobre la condición celiaca, brinda asistencia social, contención a los pacientes recién diagnosticados, colabora con los hospitales y centros de investigación relacionados con el tratamiento, da a conocer la lista de productos alimenticios industrializados aptos para celiacos

Sujetos de estudio: se incluyeron adultos celiacos de ambos sexos registrados en la FUPACEL y aceptaron responder la encuesta en línea.

Tipo de muestreo: no probabilístico por conveniencia.

Recolección de datos: los datos fueron recogidos a través de una encuesta virtual de 18 ítems, que fue adaptada de la encuesta realizada por el Defensor del Pueblo de España. La encuesta se puso a disposición de los potenciales participantes mediante un enlace que fue publicado en la página oficial de la FUPACEL y que al ingresar se les presentaba un consentimiento informado.

Análisis estadísticos: se utilizó el programa Microsoft Excel 2010. Para el análisis estadístico se utilizó el programa Epi-Info (CDC, Atlanta). Se calcularon las frecuencias relativas porcentual.

Consideraciones Éticas: El estudio fue aprobado por el comité de Ética de la Universidad del Pacifico y las autoridades a cargo de la FUPACEL. La encuesta fue anónima, voluntaria y con el consentimiento de cada encuestado.

\section{RESULTADOS}

Se incluyeron 237 adultos con enfermedad celiaca, de los cuales $70,4 \%$ era del sexo femenino, $69 \%$ tenía trabajo, la edad de diagnóstico fue en el 34,5\% entre 20 30 años (Tabla 1).

Tabla 1. Características demográficas de adultos celiacos registrados en la FUPACEL en el 2020. $n=237$

\begin{tabular}{lll}
\hline Características & $\mathbf{n}$ & $\%$ \\
\hline Sexo & & \\
Femenino & 167 & 70,4 \\
Masculino & 70 & 29,5 \\
Trabaja & 164 & 69,1 \\
Edad de diagnóstico & & \\
$1-10$ años & 30 & 12,6 \\
$11-19$ años & 33 & 14 \\
$20-30$ años & 82 & 34,5 \\
$30-40$ años & 47 & 20 \\
$>40$ años & 45 & 18,9 \\
\hline
\end{tabular}

El 95,8\% presentó alguna sintomatología en el momento del diagnóstico, siendo la distensión abdominal la más frecuente $(69,1 \%)$, seguido de diarrea crónica $(54,2 \%)$ y dolor abdominal (50\%). El 58,2\% informó alguna enfermedad asociada, el hipotiroidismo $(16,8 \%)$, anemia ferropénica $(14,3 \%)$, y dermatitis herpetiforme $(10,1 \%)$ fueron las más frecuentes. El 49,8\% informó algún familiar con EC, $9 \%$ 
hermanos, $6,3 \%$ padres, $5 \%$ hijos. El $41,8 \%$ requirió hospitalización por lo menos una vez, el 16,1\% necesitó más de 3 veces (Tabla 2 ).

Tabla 2. Características clínicas de adultos celiacos registrados en la FUPACEL en el 2020. $N=237$

\begin{tabular}{lll}
\hline Características clínicas & $\mathbf{n}$ & $\mathbf{\%}$ \\
\hline Presentó síntomas & $\mathbf{2 2 7}$ & $\mathbf{9 5 , 8}$ \\
Distensión abdominal & 163 & 69,1 \\
Diarrea crónica & 128 & 54,2 \\
Dolor abdominal & 118 & 50 \\
Caída de pelo & 105 & 44,5 \\
Pérdida de peso & 97 & 41,1 \\
Problemas en piel & 95 & 40,3 \\
No presentó síntomas & 10 & 4,2 \\
Enfermedades asociadas & $\mathbf{1 3 8}$ & $\mathbf{5 8 , 2}$ \\
\hline Hipotiroidismo & 40 & 16,8 \\
Anemia ferropénica & 34 & 14,3 \\
Dermatitis herpetiforme & 23 & 10,1 \\
Diabetes mellitus & 16 & 6,7 \\
Artritis reumatoide & 12 & 5,3 \\
Lupus & 3 & 1.3 \\
No presenta & 99 & 43,4 \\
Antecedentes familiares & $\mathbf{1 1 8}$ & $\mathbf{4 9 , 8}$ \\
\hline Tíos/primos & 40 & 17 \\
Hermanos & 21 & 9 \\
Padres & 15 & 6,3 \\
Hijos & 12 & 5 \\
No tiene & 119 & 50,2 \\
Frecuencia de hospitalizaciones & $\mathbf{9 9}$ & $\mathbf{4 1 , 8}$ \\
\hline >3 veces & 38 & 16,1 \\
2-3 veces & 30 & 13 \\
1 vez & 31 & 12,7 \\
No tuvo & 138 & 58,2 \\
\hline & & \\
\hline & &
\end{tabular}

En relación a la dieta sin gluten, el $87,3 \%$ informó que sentía que la DSG era suficiente tratamiento y el $82,7 \%$ se sentía preocupado si no podían cumplirla.

Con respecto al acceso a los productos sin gluten, el $48 \%$ podía solventar los gastos de su alimentación, $21,1 \%$ recibía ayuda de su familia, 24,9\% podían solventar el gasto solo en ocasiones, $5,9 \%$ no podían solventar los gastos. El $92 \%$ refirieron que los productos sin gluten son costosos y que se necesita una ley de apoyo económico para los pacientes con enfermedad celiaca (Tabla 3).

Tabla 3. Acceso a los productos sin gluten de pacientes celiacos registrados en la FUPACEL en el 2020. $n=237$

\begin{tabular}{lcc}
\hline Disponibilidad de los productos sin gluten & $\mathbf{n}$ & $\mathbf{\%}$ \\
\hline La dieta sin gluten es suficiente como tratamiento & 207 & 87,3 \\
Está preocupado si no cumple con la dieta sin gluten & 196 & 82,7 \\
Puede solventar los gastos & 114 & 48 \\
No puede solventar, pero la familia ayuda a solventar & 59 & 24,9 \\
Solo en ocasiones puede solventar los gastos & 50 & 21,1 \\
No puede solventar los gastos & 14 & 5,9 \\
Se siente limitado al acceso de alimentos sin gluten & 188 & 79,3 \\
Los productos sin gluten son costosos & 218 & 92 \\
Se necesita una ley de apoyo económico & 218 & 92 \\
\hline
\end{tabular}




\section{DISCUSIÓN}

En el presente estudio se incluyeron 237 pacientes de la Fundación Paraguaya de Celiacos (FUPACEL) con predominio del sexo femenino y con una importante proporción con casos familiares. La preponderancia del sexo femenino y de casos familiares es característica de la EC e informado por varios estudios ${ }^{(5-10)}$. Por tratarse de una enfermedad genética se recomienda que cuando se identifica un caso, todos los familiares consanguíneos sean evaluados buscando esta afección, que puede hallarse silente (1920). Entre los individuos con alto riesgo de presentación de EC se verifica una prevalencia de 5-15\%, que es mayor a la de la población general. En este grupo se encuentran los familiares de primer grado de pacientes celíacos (padres, hermanos e hijos) ${ }^{(20-24)}$.

La edad de diagnóstico fue en más del 30\% entre 20-30 años, mayor a la edad media de $18 \pm 10$ años, reportada por Real et al $^{(9)}$ quienes incluyeron adolescentes y adultos. En el mismo trabajo reportaron que la edad media de diagnóstico fue $21 \pm 10$ años, que muestra un hecho bastante común que es el retraso en el diagnóstico ${ }^{(23)}$. El diagnóstico depende en gran medida de la experiencia del médico y las formas clínicas de presentación, considerando que las formas silentes y atípicas son un desafío para los profesionales de la salud. Según Real et al $^{(9)}$, en Paraguay, solo $62,7 \%$ de los celiacos debutan con la clásica diarrea crónica, lo que explicaría este retraso en el diagnóstico ${ }^{(9)}$. En el presente trabajo, la diarrea crónica fue informada por el 54,2\% después de la distensión abdominal $(69,1 \%)$. La distribución de las formas clínicas de EC es semejante a otras publicaciones ${ }^{(24)}$, lo que pone de manifiesto que la enfermedad no siempre se presenta con diarrea crónica.

La enfermedad asociada más frecuente fue el hipotiroidismo $(16,8 \%)$, aanemia ferropénica $(14,3 \%)$, dermatitis herpetiforme $(10,1 \%)$ y diabetes mellitus $(6,7 \%)$. La dermatitis herpetiforme es un ejemplo claro de enfermedad cutánea inducida por el gluten, con manifestaciones extradigestivas asociadas ${ }^{(22,24)}$. La EC se puede presentar asociada a muchas otras enfermedades de diversos órganos y de diversa naturaleza, como hepáticas, neurológicas, óseas, reumatológicas, cardíacas y renales, así como a colagenosis y otros procesos varios ${ }^{(20-24)}$. Real et al $^{(9)}$ encontraron que las afecciones acompañantes más frecuentes fueron: la dermatitis herpetiforme $(33,8 \%)$, tiroiditis crónica autoinmune $(6,6 \%)$, enfermedades del colágeno $(1,1 \%)$, migraña clásica $(0,9 \%)$, síndrome de Down $(0,6 \%)$, y un solo caso de diabetes tipo 1 , vitíligo, talasemia, hipereosinofilia e hipogammaglobulinemia común. Los afectados de síndrome de Down presentan EC asociada con una frecuencia del 5-12\%. La EC está también presente en otras alteraciones genéticas como el síndrome de Turner, el síndrome de Williams, el déficit de IgA y una gran variedad de procesos de naturaleza autoinmunitaria. Los pacientes con diabetes mellitus tipo 1 presentan una frecuencia de EC asociada entre 3 a $8 \%$. Las enfermedades tiroideas se asocian con la EC con una frecuencia media en torno al 5\%, pueden cursar con hiperfunción, hipofunción o normofunción y muestran una elevada prevalencia de anticuerpos antitiroideos positivos (en torno al $25 \%$ de los casos) ${ }^{(24)}$. El tratamiento de la EC con DSG en la diabetes juvenil no es capaz de curarla, pero facilita enormemente la consecución de un mejor control metabólico, ayuda a prevenir ciertas complicaciones, mejora la calidad de vida de los pacientes y reduce notablemente las necesidades de insulina ${ }^{(25)}$. Al igual que ocurre en los diabéticos, en la mayor parte de los casos de enfermedades tiroideas, la DSG ayuda a corregir la disfunción tiroidea asociada, permite disminuir la dosis de tratamiento hormonal e incluso consigue que éste pueda retirarse ${ }^{(24)}$.

La terapia nutricional es el único tratamiento aceptado para la EC, la eliminación del gluten induce a una mejora clínica, serológica e histológica ${ }^{(15)}$. Sin embargo, la dieta es costosa, lo que puede ser una complicación para pacientes con recursos financieros limitados. El 87,3\% de los participantes del presente estudio reconoció que la dieta sin gluten era suficiente tratamiento, lo que se interpreta que no sería un problema de falta de conocimiento sino de dificultad financiera para cumplir con la dieta, pues el $82,7 \%$ se sentía preocupado no poder cumplirla debido al costo. Los pacientes que no se adhieren a la una dieta estricta pueden presentar complicaciones que incluyen deficiencias nutricionales, mayor riesgo de fracturas óseas, riesgo aumentado de 
mortalidad, y ciertos tipos de canceres ${ }^{(26)}$. Existen organizaciones sin fines de lucro que apoyan a los pacientes celiacos a tener una buena calidad de vida, sin embargo se necesitaría mayor apoyo del gobierno considerando el alto número de personas afectadas $^{(12,18)}$.

Existen múltiples factores que hacen a esta dieta difícil de llevarse: costos, accesibilidad, palatabilidad, etcétera ${ }^{(26)}$. Un estudio realizado en 2016 en Chile demostró que los alimentos sin gluten están menos disponibles, son tres veces más costosos y contienen menos proteínas y vitaminas ${ }^{(27)}$. En el trabajo de Real et al ${ }^{(10)}$, el $28 \%$ considera que no hay suficientes elecciones alimentarias para su tratamiento. En el presente estudio el $79,3 \%$ de los pacientes refirió sentirse limitado con respecto a la disponibilidad de los alimentos sin gluten y el $92 \%$ considera que los mismos son costosos, necesitando una ley nacional que apoye económicamente a las personas con enfermedad celíaca. En la investigación llevada a cabo por el Defensor del Pueblo de España ${ }^{(18)}$ los participantes afirmaron que el precio de los alimentos sin gluten era costoso $(90,97 \%)$ y un $92 \%$ de los participantes afirmaron no haber recibido ayuda alguna. De acuerdo a una revisión sistemática, la DSG es compleja, costosa, restrictiva y difícil de cumplir ${ }^{(17)}$. Estos autores no encontraron una asociación entre los factores sociodemográficos y económicos con la adherencia a DSG.

El diagnóstico de la enfermedad celíaca supone cambios en el estilo de vida, que inciden de forma directa en la calidad de esta. Una preocupación que refería la mayoría de los participantes en el cuestionario realizado por el Defensor del Pueblo está directamente relacionada con la necesidad de seguir correctamente la DSG, y en especial de poder integrarse con naturalidad en la vida social.

Evitar la ingesta de gluten de forma estricta en un país como el nuestro, como tienen que hacer los pacientes con EC, supone una drástica modificación de los hábitos de consumos alimentarios, dentro y fuera del hogar. En la aportación nutricional de carbohidratos recomendada diariamente, los productos a base de cereales con gluten suponen la principal aportación, por lo que su sustitución con productos como el arroz, las papas, el maíz o las legumbres puede no ser sencilla. Además, el uso altamente frecuente de harinas en la confección y preparación de otros alimentos y de menús hace que el riesgo de contaminación cruzada sea muy alto, principalmente en toda la actividad de restauración ${ }^{(18)}$.

A pesar de que no fue parte de esta investigación, cabe mencionar que una de las preocupaciones más frecuentemente expresadas por los afectados por EC hace referencia a las carencias o deficiencias en la información acerca de la presencia o no de gluten en los alimentos que adquieren en los comercios o en los lugares de ocio y restauración.

Las limitaciones del presente trabajo fueron el carácter transversal del estudio y la participación en forma virtual y voluntaria para responder la encuesta de los pacientes con EC. A pesar de esta limitación, el estudio presenta las características de los pacientes con EC y las preocupaciones acerca de la dificultad de la adherencia al tratamiento debido al costo y accesibilidad de los productos sin gluten.

Conflicto de intereses: Los autores declaran no tener conflicto de intereses.

Agradecimientos: A la Fundación Paraguaya de Celiacos FUPACEL en la persona de la Doctora María Elena Aguilera de Chamorro por su colaboración para la realización de las encuestas.

Fuente de financiación: No tuvo financiación externa.

Contribución de los autores: Todos los autores han contribuido de igual manera para la investigación y la redacción del presente trabajo.

\section{REFERENCI AS BI BLI OGRÁFI CAS}

1. Fasano A, Catassi C. Clinical practice. Celiac disease. N Engl J Med. 2012 Dec 20; 367(25): 2419-26.

2. Kagnoff MF. Overview and pathogenesis of celiac disease. Gastroenterology. 2005; 128(4 Suppl 1): S10-8. 
3. Goldberg AD, Allis CD, Bernstein $E$. Epigenetics: a landscape takes shape. Cell. 2007; 128(4): 635-8.

4. Husby S, Koletzko S, Korponay-Szabó $I R$, Mearin $M L$, Phillips A, Shamir R, et al. European Society for Pediatric Gastroenterology, Hepatology, and Nutrition guidelines for the diagnosis of coeliac disease. J Pediatr Gastroenterol Nutr. enero de 2012; 54(1):136-60. Erratum in: J Pediatr Gastroenterol Nutr. 2012 Apr; 54(4):572.

5. Kang JY, Kang AH, Green A, Gwee KA, Ho KY. Systematic review: worldwide variation in the frequency of coeliac disease and changes over time. Aliment Pharmacol Ther. 2013 Aug; 38(3): 22645. doi: 10.1111/apt.12373.

6. Dubé C, Rostom A, Sy R, Cranney A, Saloojee N, Garritty C, et al. The prevalence of celiac disease in averagerisk and at-risk Western European populations: a systematic review. Gastroenterology. 2005 Apr; 128 (4 Suppl 1): S57-67. doi: 10.1053/j.gastro.2005.02.014.

7. Riestra S, Fernández E, Rodrigo L, Garcia S, Ocio G. Prevalence of Coeliac disease in the general population of northern Spain. Strategies of serologic screening. Scand J Gastroenterol. 2000 Apr; 35(4): 398-402. doi: 10.1080/003655200750023967.

8. Farrell RJ, Kelly CP. Celiac sprue. N Engl J Med. 2002 J an 17; 346(3): 180-8. doi: 10.1056/NEJ Mra010852.

9. Hardy MY, Tye-Din J A. Coeliac disease: a unique model for investigating broken tolerance in autoimmunity. Clin Transl Immunology. 2016 Nov 2; 5(11): el12. doi: $10.1038 / \mathrm{cti} .2016 .58$.

10. Shannahan S, Leffler DA. Diagnosis and Updates in Celiac Disease. Gastrointest Endosc Clin N Am. 2017 Jan; 27(1): 7992. doi: 10.1016/j.giec.2016.08.011.

11. Ontiveros N, Real-Delor RE, MoraMelgem JA, Beltrán-Cárdenas CE, Figueroa-Salcido OG, Vergara-Jiménez $M$ de $J$, et al. Prevalence of Wheat/Gluten-Related Disorders and Gluten-Free Diet in Paraguay: An Online Survey-Based Study. Nutrients. 27 de enero de 2021; 13(2):396. Melissa Jeruti Enciso. Se estima que hay más de 60.000 celiacos en Paraguay y la mayoría no lo sabe. 17 de noviembre de 2015; Disponible en:

http://economiavirtual.com.py/web/pag ina-general.php?codigo $=3959$

12. Real Delor RE, Arza G, Chamorro ME, Dalles I, Ibarra Douglas A. La Enfermedad celiaca en el Paraguay. Rev Nac (Itaugua). 2011; 3(2):7-15.
13. Real Delor RE, Centurión Medina IC. Calidad de vida en adultos del Paraguay con enfermedad celiaca. Duazary: Revista internacional de Ciencias de la Salud 2018; 15(1):61-70.

14. Kupper C. Dietary guidelines and implementation for celiac disease. Gastroenterology. 2005 Apr; 128 (4 Suppl 1): S121-7. doi: 10.1053/j.gastro.2005.02.024.

15. Nasr I, Leffler DA, Ciclitira PJ. Management of celiac disease. Gastrointest Endosc Clin N Am. 2012 Oct; 22(4): 695-704. doi: 10.1016/j.giec.2012.07.012.

16. Hall NJ, Rubin G, Charnock A. Systematic review: adherence to a gluten-free diet in adult patients with coeliac disease. Aliment Pharmacol Ther. 2009 Aug 15; 30(4): 315-30. doi: $\quad \underline{10.1111 / j .1365-}$ 2036.2009.04053.x.

17. Defensor del Pueblo. Estudio sobre la situación de las personas con Enfermedad celiaca en España [Internet]. Consejería de Sanidad de la Comunidad de Madrid. Vol. 1. Madrid, España: Lettergraf, S.A; 158 Disponible en: https://www.defensordelpueblo.es/ wpcontent/uploads/2017/04/Celiaquia_201 7-1.pdf

18. Fasano A, Berti I, Gerarduzzi T, Not T, Colletti RB, Drago S, et al. Prevalence of celiac disease in at-risk and not-at-risk groups in the United States: a large multicenter study. Arch Intern Med. 2003 Feb 10; 163(3): 286-92. doi: $10.1001 /$ archinte.163.3.286.

19. Green PHR, Stavropoulos SN, Panagi SG, Goldstein SL, Mcmahon DJ, Absan $\mathrm{H}$, Neugut Al. Characteristics of adult celiac disease in the USA: results of a national survey. Am J Gastroenterol. 2001 Jan; 96(1): 126-31. doi: $\quad 10.1111 / j .1572-$ 0241.2001.03462.x.

20. Costa Gomes R, Cerqueira Maia J, Fernando Arrais R, André Nunes Jatobá C, Auxiliadora Carvalho Rocha $M$, Edinilma Felinto Brito $M$, et al. The celiac iceberg: from the clinical spectrum to serology and histopathology in children and adolescents with type 1 diabetes mellitus and Down syndrome. Scand J Gastroenterol. 2016; 51(2):178-85. doi: 10.3109/00365521.2015.1079645.

21. Laurikka P, Nurminen S, Kivelä L, Kurppa K. Extraintestinal Manifestations of Celiac Disease: Early Detection for Better Long-Term Outcomes. Nutrients. 2018 Aug 3; 10(8): 1015. doi: $10.3390 /$ nu10081015. 
22. Pelegrí Calvo C, Soriano del Castillo JM, Mañes Vinuesa J. Calidad de vida y proceso diagnóstico en celíacos adultos de la Comunidad Valenciana. Nutr Hosp. 2012 Jul-Aug; 27(4): 1293-7. doi: $10.3305 /$ nh.2012.27.4.5871.

23. Green $\mathrm{PH}$. The many faces of celiac disease: clinical presentation of celiac disease in the adult population. Gastroenterology. 2005 Apr; 128 (4 Suppl 1): S74-8. doi: 10.1053/j.gastro.2005.02.016.

24. Hansen D, Brock-Jacobsen B, Lund E, Bjørn C, Hansen LP, Nielsen C, et al. Clinical benefit of a gluten-free diet in type 1 diabetic children with screeningdetected celiac disease: a population- based screening study with 2 years' follow-up. Diabetes Care. 2006 Nov; 29 (11): 2452-6. doi: 10.2337/dc06-0990.

25. White LE, Bannerman E, Gillett PM. Coeliac disease and the gluten-free diet: a review of the burdens; factors associated with adherence and impact on health-related quality of life, with specific focus on adolescence. I Hum Nutr Diet. 2016 Oct; 29(5):593-606. doi: 10.1111/jhn.12375.

26. Estévez V, Ayala J, Vespa C, Araya M. The gluten-free basic food basket: $a$ problem of availability, cost and nutritional composition. Eur J Clin Nutr. 2016 Oct; 70(10): 1215-1217. doi: $10.1038 /$ ejen.2016.139. 